\title{
Urinary tract infections in newborns
}

\author{
Gokmen Bilgili ${ }^{1}$, Mehmet Bilgehan Yuksel $^{2}$
}

The incidence of neonatal urinary tract infection (UTI) varies from 0.1 to $1 \%$ of all infants, and its frequency is inversely proportional to birth weight [1]. Neonatal UTI presents different features than those observed in other age groups for several reasons, including higher prevalence, male sex predominance, and most importantly higher risk of urosepsis [2]. As the symptoms of a UTI in a neonate, such as irritability, poor feeding and vomiting are generalized, the clinician should have a high index of suspicion. The best correlates of infection in infants with UTI were found to be a body temperature greater than $38^{\circ} \mathrm{C}$ and uncircumcised males [3]. Escherichia coli is the most likely pathogen, but Klebsiella pneumoniae, Enterobacter spp., Enterococcus spp., Pseudomonas spp., Candida spp. and coagulasenegative staphylococci have to be considered in nosocomial UTI as well [1-3]. A positive blood culture is reported in $12.4 \%$ of cases, and nosocomial UTI is nearly twice likely to result in urosepsis compared with community-acquired UTI [2]. Bladder puncture or transurethral catheter is recommended for urine sampling in neonates to avoid urinary contamination. Parenteral antibiotic regimen, including a combination of a thirdgeneration cephalosporin or aminoglycoside with ampicillin for a total of 7-14 days should be commenced urgently in the suspicion of pyelonephritis following urine sampling for culture. Vancomycin and an aminoglycoside should be considered for nosocomial UTI. In the light of the results of sensitivity testing, a single-antibiotic coverage, if possible, should be considered [1-3]. The presence of high-grade reflux, severe urinary obstruction, and recurrent pyelonephritis mandates antibacterial prophylaxis, however its efficiency has not yet been proven [2]. Radiological studies have to be undertaken once a diagnosis of UTI has been made. An abnormal renal ultrasound is reported in around one third of cases, and vesicoureteral reflux is observed in nearly one fourth during voiding cystourethrogram (VCUG). The urine should be sterile while performing a VCUG $[2,3]$.
Gram-negative antibiotic resistance is emerging worldwide and outbreaks have occurred in neonatal units due to multi-resistant gramnegative bacteria [4]. To overcome this problem in the case of a UTI, urine cultures should be appropriately obtained, and the treatment should be tailored based on sensitivity results; antibiotic prophylaxis should be spared to aforementioned high-risk groups, while emphasizing the importance of compliance; and pediatric-specific antibiograms should be used [5].

Finally, evidence-based guidelines for the diagnosis and management of UTI in febrile infants aged less than 2 months are lacking, and urgently required [2].

\section{References}

1. Edwards MS. Postnatal bacterial infections. In Martin RJ, Fanaroff AA, Walsh MC, editors: Fanaroff and Martin's neonatal-perinatal medicine: diseases of the fetus and infant, $9^{\text {th }}$ ed. Missouri, Elsevier Mosby.2011;p 816.

2. Beetz R. Evaluation and management of urinary tract infections in the neonate. Current opinion in pediatrics. 2012;24(2):205-11.

3. Zderic SA. Urinary tract infections and vesicoureteral reflux. In Gleason CA and Devaskar SU, editors: Avery's diseases of the newborn, $9^{\text {th }}$ ed. Philadelphia, Elsevier Saunders. 2012; p 1228

4. Wickramasinghe N, Suviste J, Patel M, Gray J. Strategies to manage resistant gram-negative organisms in neonates. Archives of disease in childhood Fetal and neonatal edition. 2012;97(3):F234.

5. Edlin RS, Copp HL. Antibiotic resistance in pediatric urology. Therapeutic advances in urology. 2014;6(2):54-61. 